CALT-68-2115

hep-th/9705092

\title{
The M Theory Five-Brane and the Heterotic Stringt
}

\author{
John H. Schwarz \\ California Institute of Technology, Pasadena, CA 91125, USA
}

\begin{abstract}
Brane actions with chiral bosons present special challenges. Recent progress in the description of the two main examples - the M theory five-brane and the heterotic string - is described. Also, double dimensional reduction of the $\mathrm{M}$ theory five-brane on K3 is shown to give the heterotic string.
\end{abstract}

\section{The Bosonic Part of the Five-Brane Action}

The M theory five-brane action contains a tensor gauge field, which (in linearized approximation) has a self-dual field strength. Ref. [1] analyzed the problem of coupling a $6 \mathrm{~d}$ self-dual tensor gauge field to a metric field so as to achieve general coordinate invariance. It presented a formulation in which one direction is treated differently from the other five. At the time that work was done, the author knew of no straightforward way to make the general covariance manifest. However, shortly thereafter a paper appeared [2] that presents equivalent results using a manifestly covariant formulation [3], which we refer to as the PST formulation. In the following both approaches and their relationship are described. These results have been generalized to supersymmetric actions with local kappa symmetry [4, 5, 6], but here we will only consider the bosonic theories.

\section{The Noncovariant Formulation}

Let us denote the $6 \mathrm{~d}$ (world volume) coordinates by $\sigma^{\hat{\mu}}=\left(\sigma^{\mu}, \sigma^{5}\right)$, where $\mu=0,1,2,3,4$. The $\sigma^{5}$ direction is singled out as the one that will be treated differently from the other

\footnotetext{
${ }^{1}$ Talk presented at the Trieste Conference on Duality Symmetries in String Theory - II
} 
five. 2 The $6 \mathrm{~d}$ metric $G_{\hat{\mu} \hat{\nu}}$ contains $5 \mathrm{~d}$ pieces $G_{\mu \nu}, G_{\mu 5}$, and $G_{55}$. All formulas will be written with manifest $5 \mathrm{~d}$ general coordinate invariance. As in refs. [7, 1], we represent the self-dual tensor gauge field by a $5 \times 5$ antisymmetric tensor $B_{\mu \nu}$, and its $5 \mathrm{~d}$ curl by $H_{\mu \nu \rho}=3 \partial_{[\mu} B_{\nu \rho]}$. A useful quantity is the dual

$$
\tilde{H}^{\mu \nu}=\frac{1}{6} \epsilon^{\mu \nu \rho \lambda \sigma} H_{\rho \lambda \sigma}
$$

It was shown in ref. [1] that a class of generally covariant bosonic theories can be represented in the form $L=L_{1}+L_{2}+L_{3}$, where

$$
\begin{aligned}
L_{1} & =-\frac{1}{2} \sqrt{-G} f\left(z_{1}, z_{2}\right), \\
L_{2} & =-\frac{1}{4} \tilde{H}^{\mu \nu} \partial_{5} B_{\mu \nu}, \\
L_{3} & =\frac{1}{8} \epsilon_{\mu \nu \rho \lambda \sigma} \frac{G^{5 \rho}}{G^{55}} \tilde{H}^{\mu \nu} \tilde{H}^{\lambda \sigma} .
\end{aligned}
$$

The notation is as follows: $G$ is the $6 \mathrm{~d}$ determinant $\left(G=\operatorname{det} G_{\hat{\mu} \hat{\nu}}\right)$ and $G_{5}$ is the 5 d determinant $\left(G_{5}=\operatorname{det} G_{\mu \nu}\right)$, while $G^{55}$ and $G^{5 \rho}$ are components of the inverse $6 \mathrm{~d}$ metric $G^{\hat{\mu} \hat{\nu}}$. The $\epsilon$ symbols are purely numerical with $\epsilon^{01234}=1$ and $\epsilon^{\mu \nu \rho \lambda \sigma}=-\epsilon_{\mu \nu \rho \lambda \sigma}$. A useful relation is $G_{5}=G G^{55}$. The $z$ variables are defined to be

$$
\begin{aligned}
z_{1} & =\frac{\operatorname{tr}(G \tilde{H} G \tilde{H})}{2\left(-G_{5}\right)} \\
z_{2} & =\frac{\operatorname{tr}(G \tilde{H} G \tilde{H} G \tilde{H} G \tilde{H})}{4\left(-G_{5}\right)^{2}} .
\end{aligned}
$$

The trace only involves $5 \mathrm{~d}$ indices:

$$
\operatorname{tr}(G \tilde{H} G \tilde{H})=G_{\mu \nu} \tilde{H}^{\nu \rho} G_{\rho \lambda} \tilde{H}^{\lambda \mu}
$$

The quantities $z_{1}$ and $z_{2}$ are scalars under 5 d general coordinate transformations.

Infinitesimal parameters of general coordinate transformations are denoted $\xi^{\hat{\mu}}=\left(\xi^{\mu}, \xi\right)$. Since $5 \mathrm{~d}$ general coordinate invariance is manifest, we focus on the $\xi$ transformations only. The metric transforms in the standard way

$$
\delta_{\xi} G_{\hat{\mu} \hat{\nu}}=\xi \partial_{5} G_{\hat{\mu} \hat{\nu}}+\partial_{\hat{\mu}} \xi G_{5 \hat{\nu}}+\partial_{\hat{\nu}} \xi G_{\hat{\mu} 5} .
$$

The variation of $B_{\mu \nu}$ is given by a more complicated rule, whose origin is explained in ref. [1]:

$$
\delta_{\xi} B_{\mu \nu}=\xi K_{\mu \nu}
$$

\footnotetext{
${ }^{2}$ This is a space-like direction, but one could also choose a time-like one.
} 
where

$$
K_{\mu \nu}=2 \frac{\partial\left(L_{1}+L_{3}\right)}{\partial \tilde{H}^{\mu \nu}}=K_{\mu \nu}^{(1)} f_{1}+K_{\mu \nu}^{(2)} f_{2}+K_{\mu \nu}^{(\epsilon)}
$$

with

$$
\begin{aligned}
K_{\mu \nu}^{(1)} & =\frac{\sqrt{-G}}{\left(-G_{5}\right)}(G \tilde{H} G)_{\mu \nu} \\
K_{\mu \nu}^{(2)} & =\frac{\sqrt{-G}}{\left(-G_{5}\right)^{2}}(G \tilde{H} G \tilde{H} G \tilde{H} G)_{\mu \nu} \\
K_{\mu \nu}^{(\epsilon)} & =\epsilon_{\mu \nu \rho \lambda \sigma} \frac{G^{5 \rho}}{2 G^{55}} \tilde{H}^{\lambda \sigma},
\end{aligned}
$$

and we have defined

$$
f_{i}=\frac{\partial f}{\partial z_{i}}, \quad i=1,2
$$

Assembling the results given above, ref. [1] showed that the required general coordinate transformation symmetry is achieved if, and only if, the function $f$ satisfies the nonlinear partial differential equation 8

$$
f_{1}^{2}+z_{1} f_{1} f_{2}+\left(\frac{1}{2} z_{1}^{2}-z_{2}\right) f_{2}^{2}=1
$$

As discussed in [7], this equation has many solutions, but the one of relevance to the $\mathrm{M}$ theory five-brane is

$$
f=2 \sqrt{1+z_{1}+\frac{1}{2} z_{1}^{2}-z_{2}}
$$

For this choice $L_{1}$ can reexpressed in the Born-Infeld form

$$
L_{1}=-\sqrt{-\operatorname{det}\left(G_{\hat{\mu} \hat{\nu}}+i G_{\hat{\mu} \rho} G_{\hat{\nu} \lambda} \tilde{H}^{\rho \lambda} / \sqrt{-G_{5}}\right)} .
$$

This expression is real, despite the factor of $i$, because it is an even function of $\tilde{H}$.

\section{The PST Formulation}

In ref. [2] (using techniques developed in ref. [3]) equivalent results are described in a manifestly covariant way. To do this, the field $B_{\mu \nu}$ is extended to $B_{\hat{\mu} \hat{\nu}}$ with field strength $H_{\hat{\mu} \hat{\nu} \hat{\rho}}$. In addition, an auxiliary scalar field $a$ is introduced. The PST formulation has new gauge symmetries (described below) that allow one to choose the gauge $B_{\mu 5}=0, a=\sigma^{5}$ (and hence $\partial_{\hat{\mu}} a=\delta_{\hat{\mu}}^{5}$ ). In this gauge, the covariant PST formulas reduce to the ones given above. 
Equation (12) expresses $L_{1}$ in terms of the determinant of the $6 \times 6$ matrix

$$
M_{\hat{\mu} \hat{\nu}}=G_{\hat{\mu} \hat{\nu}}+i \frac{G_{\hat{\mu} \rho} G_{\hat{\nu} \lambda}}{\sqrt{-G G^{55}}} \tilde{H}^{\rho \lambda} .
$$

In the PST approach this is extended to the manifestly covariant form

$$
M_{\hat{\mu} \hat{\nu}}^{\mathrm{cov} .}=G_{\hat{\mu} \hat{\nu}}+i \frac{G_{\hat{\mu} \hat{\rho}} G_{\hat{\nu} \hat{\lambda}}}{\sqrt{-G(\partial a)^{2}}} \tilde{H}_{\mathrm{cov} .}^{\hat{\rho} \hat{\lambda}} .
$$

The quantity

$$
(\partial a)^{2}=G^{\hat{\mu} \hat{\nu}} \partial_{\hat{\mu}} a \partial_{\hat{\nu}} a
$$

reduces to $G^{55}$ upon setting $\partial_{\hat{\mu}} a=\delta_{\hat{\mu}}^{5}$, and

$$
\tilde{H}_{\text {cov. }}^{\hat{\rho} \hat{\lambda}} \equiv \frac{1}{6} \epsilon^{\hat{\rho} \hat{\lambda} \hat{\mu} \hat{\nu} \hat{\sigma} \hat{\tau}} H_{\hat{\mu} \hat{\nu} \hat{\sigma}} \partial_{\hat{\tau}} a
$$

reduces to $\tilde{H}^{\rho \lambda}$. Thus $M_{\hat{\mu} \hat{\nu}}^{\text {cov. }}$ replaces $M_{\hat{\mu} \hat{\nu}}$ in $L_{1}$. Furthermore, the expression

$$
L^{\prime}=-\frac{1}{4(\partial a)^{2}} \tilde{H}_{\text {cov. }}^{\hat{\mu} \hat{\nu}} H_{\hat{\mu} \hat{\nu} \hat{\rho}} G^{\hat{\rho} \hat{\lambda}} \partial_{\hat{\lambda}} a
$$

which transforms under general coordinate transformations as a scalar density, reduces to $L_{2}+L_{3}$ upon gauge fixing. It is interesting that $L_{2}$ and $L_{3}$ are unified in this formulation.

Let us now describe the new gauge symmetries of ref. [2]. Since degrees of freedom $a$ and $B_{\mu 5}$ have been added, corresponding gauge symmetries are required. One of them is

$$
\delta B_{\hat{\mu} \hat{\nu}}=2 \phi_{[\hat{\mu}} \partial_{\hat{\nu}]} a
$$

where $\phi_{\hat{\mu}}$ are infinitesimal parameters, and the other fields do not vary. In terms of differential forms, this implies $\delta H=d \phi \wedge d a$. $\tilde{H}_{\text {cov. }}^{\hat{\rho} \hat{\lambda}}$ is invariant under this transformation, since it corresponds to the dual of $H \wedge d a$, but $d a \wedge d a=0$. Thus the covariant version of $L_{1}$ is invariant under this transformation. The variation of $L^{\prime}$, on the other hand, is a total derivative.

The second local symmetry involves an infinitesimal scalar parameter $\varphi$. The transformation rules are $\delta G_{\hat{\mu} \hat{\nu}}=0, \delta a=\varphi$, and

$$
\delta B_{\hat{\mu} \hat{\nu}}=\frac{1}{(\partial a)^{2}} \varphi H_{\hat{\mu} \hat{\nu} \hat{\rho}} G^{\hat{\rho} \hat{\lambda}} \partial_{\hat{\lambda}} a+\varphi V_{\hat{\mu} \hat{\nu}}
$$

where the quantity $V_{\hat{\mu} \hat{\nu}}$ is to be determined. Rather than derive it from scratch, let's see what is required to agree with the previous formulas after gauge fixing. In other words, we 
fix the gauge $\partial_{\hat{\mu}} a=\delta_{\hat{\mu}}^{5}$ and $B_{\mu 5}=0$, and figure out what the resulting $\xi$ transformations are. We need

$$
\delta a=\varphi+\xi \partial_{5} a=\varphi+\xi=0
$$

which tells us that $\varphi=-\xi$. Then

$$
\begin{aligned}
\delta_{\xi} B_{\mu \nu} & =\frac{1}{(\partial a)^{2}} \varphi H_{\mu \nu \hat{\rho}} G^{\hat{\rho} \hat{\lambda}} \partial_{\hat{\lambda}} a+\varphi V_{\mu \nu}+\xi H_{5 \mu \nu} \\
& =-\xi\left(\frac{G^{\rho 5}}{G^{55}} H_{\mu \nu \rho}+V_{\mu \nu}\right)=\xi\left(K_{\mu \nu}^{(\epsilon)}-V_{\mu \nu}\right)
\end{aligned}
$$

Thus, comparing with eqs. (6) and (7), we need the covariant definition

$$
V_{\hat{\mu} \hat{\nu}}=-2 \frac{\partial L_{1}}{\partial \tilde{H}_{\mathrm{cov}}^{\hat{\mu} \hat{\nu}}}
$$

to achieve agreement with our previous results.

\section{A New Heterotic String Action}

There are two main approaches to constructing the world-sheet action of the heterotic string that have been used in the past [9]. In one of them, the internal torus is described in terms of bosonic coordinates. The fact that these bosons are chiral (i.e., the left-movers and rightmovers behave differently) is imposed through external constraints. In the second approach these bosonic coordinates are replaced by world-sheet fermions, which are Majorana-Weyl in the $2 \mathrm{~d}$ sense. What will be most convenient for our purposes is a variant of the first approach. In this variant the coordinates of the Narain torus are still represented by bosonic fields, but the chirality of these fields is achieved through new gauge invariances rather than external constraints [10].

Consider the Narain compactified heterotic string in a Minkowski space-time with $d=$ $10-n$ dimensions [11]. Let these coordinates be denoted by $X^{m}$ with $m=0,1, \ldots, d-1=$ $9-n$. To properly account for all the degrees of freedom, the Narain torus should be described by $16+2 n$ bosonic coordinates $Y^{I}, I=1,2, \ldots, 16+2 n$. These will be arranged to describe $26-d=16+n$ left-movers and $10-d=n$ right-movers. The $Y^{I}$ are taken to be angular coordinates, with period $2 \pi$, so that $Y^{I} \sim Y^{I}+2 \pi$, and the conjugate momenta are integers. The actual size and shape of the torus is encoded in a matrix of moduli, denoted $M_{I J}$, which will be described below. 
The $(16+2 n)$-dimensional lattice of allowed momenta should form an even self-dual lattice of signature $(n, 16+n)$. Let us therefore introduce a matrix

$$
\eta=\left(\begin{array}{cc}
I_{n} & 0 \\
0 & -I_{16+n}
\end{array}\right),
$$

where $I_{n}$ is the $n \times n$ unit matrix. An even self-dual lattice with this signature has a set of $16+2 n$ basis vectors $V_{I}$, and the symmetric matrix

$$
L_{I J}=V_{I}^{a} \eta_{a b} V_{J}^{b}
$$

characterizes the lattice. A convenient specific choice is

$$
L=\Lambda_{8} \oplus \Lambda_{8} \oplus \sigma \oplus \ldots \oplus \sigma
$$

where $\Lambda_{8}$ is the negative of the $E_{8}$ Cartan matrix and $\sigma=\left(\begin{array}{ll}0 & 1 \\ 1 & 0\end{array}\right)$ appears $n$ times.

The Narain moduli space is characterized, up to $T$ duality equivalences that will be discussed below, by a symmetric matrix $M_{a b}^{\prime} \in O(n, 16+n)$, which satisfies $M^{\prime} \eta M^{\prime}=\eta$. The fact that it is symmetric means that it actually parametrizes the coset space $O(n, 16+$ $n) / O(n) \times O(16+n)$, which has $n(16+n)$ real dimensions. To describe the $T$ duality equivalences, it is convenient to change to the basis defined by the basis vectors of the self-dual lattice. Accordingly, we define

$$
M_{I J}=V_{I}^{a} M_{a b}^{\prime} V_{J}^{b}=\left(V^{T} M^{\prime} V\right)_{I J}
$$

This matrix is also symmetric and satisfies

$$
M L^{-1} M=L
$$

from which it follows that $\left(L^{-1} M\right)^{2}=1$. This allows us to define projection operators

$$
\mathcal{P}_{ \pm}=\frac{1}{2}\left(1 \pm L^{-1} M\right)
$$

$\mathcal{P}_{+}$projects onto an $n$-dimensional subspace, which will correspond to right-movers. Similarly, $\mathcal{P}_{-}$projects onto the $(16+n)$-dimensional space of left-movers. The theory we are seeking should be invariant under an infinite discrete group of $T$ duality transformations, denoted $\Gamma_{n, 16+n}$, s so that the actual moduli space is the standard Narain space

$$
\mathcal{M}_{n, 16+n}=\Gamma_{n, 16+n} \backslash O(n, 16+n) / O(n) \times O(16+n) .
$$

\footnotetext{
${ }^{3}$ It is often called $O(n, 16+n ; \mathbf{Z})$.
} 
The desired equations of motion for the $Y$ coordinates are [12, 13, 14, 15]

$$
\mathcal{P}_{-} \partial_{+} Y=0 \text { and } \mathcal{P}_{+} \partial_{-} Y=0
$$

where $\xi^{ \pm}=\xi^{1} \pm \xi^{0}$, so that $\partial_{ \pm}=\frac{1}{2}\left(\partial_{1} \pm \partial_{0}\right) . \xi^{0}$ and $\xi^{1}$ are the world-sheet time and space, respectively. The pair of equations in (30) can be combined in the form

$$
M \partial_{0} Y-L \partial_{1} Y=0
$$

It is easy to write down a lagrangian that gives this equation [16]:

$$
\mathcal{L}_{N}=\frac{1}{2}\left(\partial_{0} Y M \partial_{0} Y-\partial_{0} Y L \partial_{1} Y\right)
$$

Two things are peculiar about this lagrangian. First, it does not have manifest Lorentz invariance. However, in ref. [17] it was shown that $\mathcal{L}_{N}$ has a global symmetry that can be interpreted as describing a non-manifest Lorentz invariance. Second, it gives the equation of motion

$$
\partial_{0}\left[M \partial_{0} Y-L \partial_{1} Y\right]=0
$$

which has a second, unwanted, solution $Y^{I}=f^{I}\left(\xi^{1}\right)$. The resolution of the second problem is quite simple. The transformation $\delta Y^{I}=f^{I}\left(\xi^{1}\right)$ is a gauge symmetry of $\mathcal{L}_{N}$, and therefore $f^{I}\left(\xi^{1}\right)$ represents unphysical gauge degrees of freedom.

The first problem, the noncovariance of $\mathcal{L}_{N}$, is more interesting. We will follow the PST approach [3], and extend $\mathcal{L}_{N}$ to a manifestly Lorentz invariant action by introducing an auxiliary scalar field $a(\xi)$. The desired generalization of $\mathcal{L}_{N}$ is then

$$
\mathcal{L}_{P S T}=\frac{1}{2(\partial a)^{2}}(\tilde{Y} M \tilde{Y}-\tilde{Y} L \partial Y \cdot \partial a)
$$

where

$$
\tilde{Y}^{I}=\epsilon^{\alpha \beta} \partial_{\alpha} Y^{I} \partial_{\beta} a
$$

Also, $(\partial a)^{2}$ and $\partial Y \cdot \partial a$ are formed using the $2 \mathrm{~d}$ Lorentz metric, which is diagonal with $\eta^{00}=-1$ and $\eta^{11}=1$.

The theory given by $\mathcal{L}_{P S T}$ has two gauge invariances. The first is

$$
\begin{aligned}
\delta Y & =\varphi\left(\frac{1}{\partial_{+} a} \mathcal{P}_{-} \partial_{+} Y+\frac{1}{\partial_{-} a} \mathcal{P}_{+} \partial_{-} Y\right), \\
\delta a & =\varphi
\end{aligned}
$$


where $\varphi\left(\xi^{0}, \xi^{1}\right)$ is an arbitrary infinitesimal scalar function. If this gauge freedom is used to set $a=\xi^{1}$, then $\mathcal{L}_{P S T}$ reduces to $\mathcal{L}_{N}$. The second gauge invariance is

$$
\delta Y^{I}=f^{I}(a), \quad \delta a=0
$$

where $f^{I}$ are arbitrary infinitesimal functions of one variable. This is the covariant version of the gauge symmetry of $\mathcal{L}_{N}$ that was used to argue that the undesired solution of the equations of motion is pure gauge.

\section{Reparametrization Invariant Action}

The formulas described above are not the whole story of the bosonic degrees of freedom of the toroidally compactified heterotic string, because they lack the Virasoro constraint conditions. The standard way to remedy this situation is to include an auxiliary world-sheet metric field $g_{\alpha \beta}(\xi)$, so that the world-sheet Lorentz invariance is replaced by world-sheet general coordinate invariance. Since we now want to include the coordinates $X^{m}$ describing the uncompactified dimensions, as well, let us also introduce an induced world-sheet metric

$$
G_{\alpha \beta}=g_{m n}(X) \partial_{\alpha} X^{m} \partial_{\beta} X^{n}
$$

where $g_{m n}(X)$ is the string frame target-space metric in $d$ dimensions. It is related to the canonically normalized metric by a factor of the form $\exp (\alpha \phi)$, where $\phi$ is the dilaton and $\alpha$ is a numerical constant, which can be computed by requiring that the target-space lagrangian is proportional $\exp (-2 \phi)$. We will mostly be interested in taking $\phi$ to be a constant and $g_{m n}$ to be proportional to the flat Minkowski metric. Then the heterotic string coupling constant is $\lambda_{H}=\exp \phi$, and the desired world sheet lagrangian is

$$
\mathcal{L}_{g}=-\frac{1}{2} \sqrt{-g} g^{\alpha \beta} G_{\alpha \beta}+\frac{\tilde{Y} M \tilde{Y}}{2 \sqrt{-g}(\partial a)^{2}}-\frac{\tilde{Y} L \partial Y \cdot \partial a}{2(\partial a)^{2}} .
$$

Now, of course, $(\partial a)^{2}=g^{\alpha \beta} \partial_{\alpha} a \partial_{\beta} a$ and $\partial Y \cdot \partial a=g^{\alpha \beta} \partial_{\alpha} Y \partial_{\beta} a$. The placement of the $\sqrt{-g}$ factors reflects the fact that $\tilde{Y} / \sqrt{-g}$ transforms as a scalar.

There are a few points to be made about $\mathcal{L}_{g}$. First of all, the PST gauge symmetries continue to hold, so it describes the correct degrees of freedom. Second, just as for more conventional string actions, it has Weyl invariance: $g_{\alpha \beta} \rightarrow \lambda g_{\alpha \beta}$ is a local symmetry. This ensures that the stress tensor

$$
T_{\alpha \beta}=-\frac{2}{\sqrt{-g}} \frac{\delta S_{g}}{\delta g^{\alpha \beta}}
$$


is traceless $\left(g^{\alpha \beta} T_{\alpha \beta}=0\right)$. Using the general coordinate invariance to choose $g_{\alpha \beta}$ conformally flat, and using the PST gauge invariance to set $a=\xi^{1}$, the $Y$ equations of motion reduce to those described in the previous subsection. In addition, one obtains the classical Virasoro constraints $T_{++}=T_{--}=0$.

The lagrangian $\mathcal{L}_{g}$ is written with an auxiliary world-volume metric, which is called the Howe-Tucker or Polyakov formulation. This is the most convenient description for many purposes. However, for the purpose of comparing to expressions derived from the M5-brane later in this paper, it will be useful to also know the version of the lagrangian in which the auxiliary metric is eliminated - the Nambu-Goto formulation. Note that $\mathcal{L}_{g}$ only involves the metric components in the combination $\sqrt{-g} g^{\alpha \beta}$, which has two independent components. It is a straightforward matter to solve their equations of motion and eliminate them from the action. This leaves the final form for the bosonic part of the heterotic string in $10-n$ dimensions

$$
\mathcal{L}=-\sqrt{-G} \sqrt{1+\frac{\tilde{Y} M \tilde{Y}}{G(\partial a)^{2}}+\left(\frac{\tilde{Y} L \tilde{Y}}{2 G(\partial a)^{2}}\right)^{2}}-\frac{\tilde{Y} L \partial Y \cdot \partial a}{2(\partial a)^{2}}
$$

where $G=\operatorname{det} G_{\alpha \beta}$, and now

$$
(\partial a)^{2}=G^{\alpha \beta} \partial_{\alpha} a \partial_{\beta} a, \quad \partial Y \cdot \partial a=G^{\alpha \beta} \partial_{\alpha} Y \partial_{\beta} a .
$$

\section{$3 \quad$ Wrapping the M-Theory Five-Brane on K3}

Let us now consider double dimensional reduction of the M5-brane on K3. Th This is supposed to give the heterotic string in seven dimensions [19, 20, 21. Our starting point is the bosonic part of the M5-brane action [7] in the general coordinate invariant PST formulation. Since the other 11d fields are still assumed to vanish, $g_{M N}(X)$ must be Ricci flat. We will take it to be a product of a Ricci-flat K3 and a flat 7d Minkowski space-time.

Since the M5-brane is taken to wrap the spatial K3, the diffeomorphism invariances of the M5-brane action in these dimensions can be used to equate the four world-volume coordinates that describe the K3 with the four target-space coordinates that describe the K3. In other words, we set $\sigma^{\mu}=\left(\xi^{\alpha}, x^{i}\right)$ and $X^{M}=\left(X^{m}, x^{i}\right)$. Note that Latin indices $i, j, k$ are used for the K3 dimensions $\left(x^{i}\right)$ and early Greek letters for the directions $\left(\xi^{\alpha}\right)$, which are

\footnotetext{
${ }^{4}$ See ref. [18] for a review of the mathematics of $\mathrm{K} 3$ and some of its appearances in string theory dualities.
} 
the world-sheet coordinates of the resulting string action. This wrapping by identification of coordinates, together with the extraction of the K3 zero modes, is what is meant by double dimensional reduction. With these choices, the $6 \mathrm{~d}$ metric can be decomposed into blocks

$$
\left(G_{\mu \nu}\right)=\left(\begin{array}{cc}
\tilde{G}_{\alpha \beta} & 0 \\
0 & h_{i j}
\end{array}\right)
$$

with $h_{i j}$ and $\tilde{G}_{\alpha \beta}$ being the K3 metric and the induced metric on the string world-sheet, respectively. The purpose of the tilde is to emphasize that $\tilde{G}_{\alpha \beta}=\tilde{g}_{m n} \partial_{\alpha} X^{m} \partial_{\beta} X^{n}$, where $\tilde{g}_{m n}$ is the $7 \mathrm{~d}$ part of the canonical $11 \mathrm{~d}$ metric. It differs from the metric introduced earlier by a scale factor, which will be determined below. It is convenient to take the PST scalar field $a$ to depend on the $\xi^{\alpha}$ coordinates only. This amounts to partially fixing a gauge choice for the PST gauge invariance.

The two-form field $B$ has the following contributions from K3 zero modes:

$$
B_{i j}=\sum_{I=1}^{22} Y^{I}(\xi) b_{I i j}(x), \quad B_{\alpha i}=0, \quad B_{\alpha \beta}=c_{\alpha \beta}(\xi)
$$

where $b_{I i j}$ are the 22 harmonic representatives of $\mathrm{H}^{2}(\mathrm{~K} 3, \mathbf{Z})$, the integral second cohomology classes of K3. Any other terms are either massive or can be removed by gauge transformations. The nonzero components of $H_{\mu \nu \rho}$ and $\tilde{H}^{\mu \nu}$ are

$$
\begin{gathered}
H_{\alpha i j}=\sum_{I=1}^{22} \partial_{\alpha} Y^{I} b_{I i j} \\
\tilde{H}^{i j}=\sum_{I=1}^{22} \tilde{Y}^{I} \frac{1}{2} \epsilon^{i j k l} b_{I k l}=\sum_{I=1}^{22} \sqrt{h} \tilde{Y}^{I}\left(* b_{I}\right)^{i j},
\end{gathered}
$$

where $\tilde{Y}^{I}=\epsilon^{\alpha \beta} \partial_{\alpha} Y^{I} \partial_{\beta} a$ as in eq. (35). Note that $c_{\alpha \beta}$ does not contribute.

Now we can compute the string action that arises from double dimensional reduction by substituting the decompositions (45) and (46) into the five-brane Lagrangian. To make the connection with the heterotic string action of the previous section, we make the identifications

$$
\begin{gathered}
L_{I J}=\int_{K 3} b_{I} \wedge b_{J}, \\
M_{I J}=\int_{K 3} b_{I} \wedge * b_{J} .
\end{gathered}
$$


Note that $* b_{I}=b_{J}\left(L^{-1} M\right)_{I}^{J}$, and therefore $\left(L^{-1} M\right)^{2}=1$, as in sect. 2. Note also that $b_{I} \wedge b_{J}$ and $b_{I} \wedge * b_{J}$ are closed four-forms, and therefore they are cohomologous to the unique harmonic four-form of the K3, which is the volume form $\omega$. It follows that

$$
b_{I} \wedge b_{J}=* b_{I} \wedge * b_{J}=\frac{L_{I J}}{\mathcal{V}} \omega+d T_{I J}, \quad b_{I} \wedge * b_{J}=\frac{M_{I J}}{\mathcal{V}} \omega+d U_{I J},
$$

where $\mathcal{V}=\int_{K 3} \omega$ is the volume of the $\mathrm{K} 3$ and $U_{I J}=T_{I K}\left(L^{-1} M\right)^{K}{ }_{J}$. The exact terms are absent when either two-form is self-dual, but there is no apparent reason why they should vanish when both of them are anti-self-dual. If we nevertheless ignore the exact pieces in these formulas, substitute into the Lagrangian, and integrate over the K3, we obtain

$$
\mathcal{L}_{1}=-\mathcal{V} \sqrt{-\tilde{G}} \sqrt{1+\frac{\tilde{Y}^{I} M_{I J} \tilde{Y}^{J}}{\tilde{G}(\partial a)^{2} \mathcal{V}}+\frac{1}{4}\left(\frac{\tilde{Y}^{I} L_{I J} \tilde{Y}^{J}}{\tilde{G}(\partial a)^{2} \mathcal{V}}\right)^{2}}-\frac{\tilde{Y}^{I} L_{I J} \partial_{\alpha} Y^{J} \tilde{G}^{\alpha \beta} \partial_{\beta} a}{2(\partial a)^{2}}
$$

This is precisely the heterotic string lagrangian (for $n=3$ ) presented in eq. (41) of the previous section provided that the $7 \mathrm{~d}$ metric $g_{m n}$ in the string frame is related to the metric $\tilde{g}_{m n}$ derived from $11 \mathrm{~d}$ by

$$
g_{m n}=\mathcal{V} \tilde{g}_{m n}
$$

so that $G_{\alpha \beta}=\mathcal{V} \tilde{G}_{\alpha \beta}$. This is the same scaling rule found by a different argument in ref. [19]. Then, following ref. [19], the Einstein term in the $7 \mathrm{~d}$ lagrangian is proportional to $\mathcal{V} \sqrt{-\tilde{g}} R(\tilde{g})=\mathcal{V}^{-3 / 2} \sqrt{-g} R(g)$, from which one infers that $\mathcal{V} \sim \lambda_{H}^{4 / 3}$.

To complete the argument we must still explain why terms that have been dropped make negligible contributions. It is not at all obvious that the exact pieces in eq. (49) can be neglected, but it is what is required to obtain the desired answer. The other class of terms that have been dropped are the Kaluza-Klein excitations of the five-brane on the K3. By simple dimensional analysis, one can show that in the heterotic string metric these contributions to the mass-squared of excitations are of order $\lambda_{H}^{-2}$. Therefore they represent non-perturbative corrections from the heterotic viewpoint. Since our purpose is only to reproduce the perturbative heterotic theory, they can be dropped. Another class of contributions, which should not be dropped, correspond to simultaneously wrapping the M2-brane around a 2-cycle of the K3. These wrappings introduce charges for the $22 \mathrm{U}(1)$ 's, according to how many times each cycle is wrapped. The contribution to the mass-squared of excitations depends on the shape of the K3, of course, but in the heterotic metric it is independent of its volume and hence of the heterotic string coupling constant. 


\section{Acknowledgment}

I am grateful to M. Aganagic, S. Cherkis, J. Park, M. Perry, and C. Popescu for collaborating on portions of this work. This work is supported in part by the U.S. Dept. of Energy under Grant No. DE-FG03-92-ER40701.

\section{References}

[1] J.H. Schwarz, "Coupling a Self-Dual Tensor to Gravity in Six Dimensions," hepth/9701008.

[2] P. Pasti, D. Sorokin, and M. Tonin, "Covariant Action for a D=11 Five-Brane with the Chiral Field," hep-th/9701037.

[3] P. Pasti, D. Sorokin, and M. Tonin, Phys. Rev. D52 (1995) 4277, hep-th/9506109; "On Lorentz Invariant Actions for Chiral P-Forms," hep-th/9611100.

[4] I. Bandos, K. Lechner, A. Nurmagambetov, P. Pasti, D. Sorokin, and M. Tonin, "Covariant Action for the Super-Five-Brane of M-Theory," hep-th/9701149.

[5] M. Aganagic, J. Park, C. Popescu, and J. H. Schwarz, "World-Volume Action of the M Theory Five-Brane, "hep-th/9701166.

[6] P.S. Howe, E. Sezgin, P.C. West, "Covariant Field Equations of the M Theory FiveBrane," hep-th/9702008.

[7] M. Perry and J.H. Schwarz, "Interacting Chiral Gauge Fields in Six Dimensions and Born-Infeld Theory," hep-th/9611065.

[8] G.W. Gibbons and D.A. Rasheed, Nucl. Phys. B454 (1995) 185, hep-th/9506035.

[9] D.J. Gross, J.A. Harvey, E. Martinec, and R. Rohm, Nucl. Phys. B256 (1985) 253.

[10] S. Cherkis and J.H. Schwarz, "Wrapping the M Theory Five-brane on K3," hepth/9703062.

[11] K.S. Narain, Phys. Lett. B169 (1986) 41; K.S. Narain, M.H. Sarmadi, and E. Witten Nucl. Phys. B279 (1987) 369.

[12] S. Cecotti, S. Ferrara, and L. Girardello, Nucl. Phys. B308 (1988) 436. 
[13] M. Duff, Nucl. Phys. B335 (1990) 610.

[14] A. Tseytlin, Phys. Lett. B242 (1990) 163; Nucl. Phys. B350 (1991) 395; Phys. Rev. Lett. 66 (1991) 545.

[15] J. Maharana and J.H. Schwarz, Nucl. Phys. B390 (1993) 3, hep-th/9207016.

[16] R. Floreanini and R. Jackiw, Phys. Rev. Lett. 59 (1987) 1873.

[17] J.H. Schwarz and A. Sen, Nucl. Phys. B411 (1994) 35, hep-th/9304154.

[18] P.S. Aspinwall, "K3 Surfaces and String Duality," hep-th/9611137.

[19] E. Witten, Nucl. Phys. B443 (1995) 85, hep-th/9503124.

[20] J.A. Harvey and A. Strominger, Nucl. Phys. B449 (1995) 535, hep-th/9504047; A. Sen, Nucl. Phys. B450 (1995) 103, hep-th/9504027.

[21] P.K. Townsend, Phys. Lett. B354 (1995) 247, hep-th/9504095. 